University of Nebraska - Lincoln

DigitalCommons@University of Nebraska - Lincoln

Faculty Publications from the Department of Electrical \& Computer Engineering, Department Electrical and Computer Engineering

2009

\title{
Controlled growth of carbon nanotubes on electrodes under different bias polarity
}

Yi Gao

University of Nebraska-Lincoln, ygao3@Unl.edu

Yunshen Zhou

University of Nebraska-Lincoln, yzhou5@unl.edu

W. Xiong

University of Nebraska-Lincoln

M. Mahjouri-Samani

University of Nebraska-Lincoln

M. Mitchell

University of Nebraska-Lincoln

See next page for additional authors

Follow this and additional works at: https://digitalcommons.unl.edu/electricalengineeringfacpub

Part of the Electrical and Computer Engineering Commons

Gao, Yi; Zhou, Yunshen; Xiong, W.; Mahjouri-Samani, M.; Mitchell, M.; and Lu, Yongfeng, "Controlled growth of carbon nanotubes on electrodes under different bias polarity" (2009). Faculty Publications from the Department of Electrical and Computer Engineering. 116.

https://digitalcommons.unl.edu/electricalengineeringfacpub/116

This Article is brought to you for free and open access by the Electrical \& Computer Engineering, Department of at DigitalCommons@University of Nebraska - Lincoln. It has been accepted for inclusion in Faculty Publications from the Department of Electrical and Computer Engineering by an authorized administrator of DigitalCommons@University of Nebraska - Lincoln. 


\section{Authors}

Yi Gao, Yunshen Zhou, W. Xiong, M. Mahjouri-Samani, M. Mitchell, and Yongfeng Lu 


\title{
Controlled growth of carbon nanotubes on electrodes under different bias polarity
}

\author{
Y. Gao, Y. S. Zhou, W. Xiong, M. Mahjouri-Samani, M. Mitchell, and Y. F. Lu ${ }^{\text {a) }}$ \\ Department of Electrical Engineering, University of Nebraska-Lincoln, Lincoln, Nebraska 68588-0511, USA
}

(Received 25 July 2009; accepted 18 September 2009; published online 8 October 2009)

\begin{abstract}
Carbon nanotubes (CNTs) of different alignments, such as surface-bounded and vertically aligned arrays, enable applications in different fields. In this study, controlled growth of CNTs with different alignments was achieved by electrically biasing catalyzed electrodes with different polarities in a laser-assisted chemical vapor deposition process. CNT growth was suggested to be guided by the movement of electrically charged catalyst-nanoparticles under the influence of an external electric field. This discovery provides a convenient approach to control the alignment of CNT arrays for different applications. (C) 2009 American Institute of Physics. [doi:10.1063/1.3246144]
\end{abstract}

Carbon nanotubes (CNTs) of different alignments yield applications in different fields. ${ }^{1-6}$ Growth of CNTs with controlled alignments is a key prerequisite for developing CNTbased devices. ${ }^{1,2}$ Extensive investigations have been made on controllable growth of CNTs with different alignments, including template-assisted, ${ }^{5,6}$ gas-flow induced, ${ }^{7}$ fast heating induced, ${ }^{8}$ and electric field (E-field) induced alignments. ${ }^{3,9}$ E-field induced aligned growth of CNTs ${ }^{10-16}$ has been investigated and applied in fabrication of ordered CNT arrays. However, mechanisms suggested to explain the E-field alignment effects are still controversial. Zhang et al. ${ }^{3}$ suggested that torque on the dipole moment along the CNT axis induced by the E-field overwhelmed both thermal fluctuation and gas flow and dominated the alignment of CNTs. Bao and $\mathrm{Pan}^{15}$ claimed that the electrostatic force on the catalyst particles enabled the aligned growth of CNTs.

In this study, controlled growth of CNTs with different alignments was achieved by applying electrical biases of different polarities on electrodes during CNT growth by laser-assisted chemical vapor deposition (LCVD). Surfacebounded CNTs were found to crawl out from anodes while vertically aligned CNTs were found to dominate the cathodes. It was suggested that the selective growth of CNTs with different alignments was guided by the movement of positively charged catalyst nanoparticles (NPs) in the external E-fields. The surface-bounded CNTs on the anodes were ascribed to the repulsive force between the catalyst NPs and the anodes, while the vertically aligned CNTs were ascribed to the joint interactions from the attractive force between the catalyst NPs and the cathodes as well as the strong interactions among the CNTs.

CNTs were synthesized on prepatterned $\mathrm{Si} / \mathrm{SiO}_{2}$ substrates using the LCVD method. ${ }^{1,2}$ Figure 1(a) shows the schematic experimental setup. Figure 1(b) shows a typical $\mathrm{Si} / \mathrm{SiO}_{2}$ substrate prepatterned with metallic electrodes. Heavily doped $\mathrm{Si}$ wafers covered with a $2 \mu \mathrm{m}$ thick $\mathrm{SiO}_{2}$ layer were used as the substrates. The electrodes, including point-to-point and cross-shaped patterns, were fabricated by optical lithography, followed by dc sputtering. Ruthenium $(\mathrm{Ru})$ films of $180 \mathrm{~nm}$ thick were deposited as the electrodes

\footnotetext{
a) Author to whom correspondence should be addressed. Tel.: (402) 4728323. FAX: (402) 472-4732. Electronic mail: ylu2@unl.edu. URL: http:// lane.unl.edu.
}

due to its low solubility with $\mathrm{Fe}$, high work function, and high melting point. Tri-layer Al/Fe/Al $(2 / 1 / 1 \mathrm{~nm})$ catalysts were deposited on the Ru electrodes. The CNT growth was carried out in an LCVD chamber. A continuous-wave $\mathrm{CO}_{2}$ laser (synrad, firestar v40, wavelength of $10.6 \mu \mathrm{m}$ ) was used to irradiate the sample. The reaction temperature was controlled to be around $500 \pm 20{ }^{\circ} \mathrm{C}$. A dc voltage was applied to bias the electrodes and control the aligned growth of CNTs. A gas mixture of acetylene $\left(\mathrm{C}_{2} \mathrm{H}_{2}\right)$ and anhydrous ammonia $\left(\mathrm{NH}_{3}\right)$ with a volume ratio of $1: 10$ was introduced into the chamber at a pressure of 10 Torr. $\mathrm{NH}_{3}$ is used as a buffer gas to dilute $\mathrm{C}_{2} \mathrm{H}_{2}$, creating an etching environment to suppress the growth of amorphous carbon and protect catalyst particles from being poisoned by amorphous carbon. ${ }^{17}$ The reaction time was approximately $1.5 \mathrm{~min}$.

A field-emission scanning electron microscope (Hitachi S4700 FE-SEM system, maximum resolution of $1.2 \mathrm{~nm}$ at $25 \mathrm{kV}$ ) was used to observe the CNTs. A Renishaw inVia dispersive micro-Raman spectrometer was used for Raman spectroscopy. A standard $50 \times$ objective was used to provide a focal spot of $\sim 2 \mu \mathrm{m}$ and a power density of $\sim 2551 \mathrm{~W} / \mathrm{cm}^{2}$. An argon-ion laser operated at $488 \mathrm{~nm}$ was used as the excitation source.

CNTs with different alignments were achieved on the metallic electrodes. As shown in Fig. 2(a), densely packed CNTs were observed both on the anode and cathode. Surface-bounded CNTs were observed on the anode sticking out from the electrode and crawling on the $\mathrm{SiO}_{2}$ surface. A fuzzy edge was observed for the anode. However, vertically aligned CNTs were observed on the cathode. A neat and clean edge was observed for the cathode. Raman spectra were measured on the anode and cathode separately, as shown in Figs. 2(b) and 2(c). The radial breathing mode
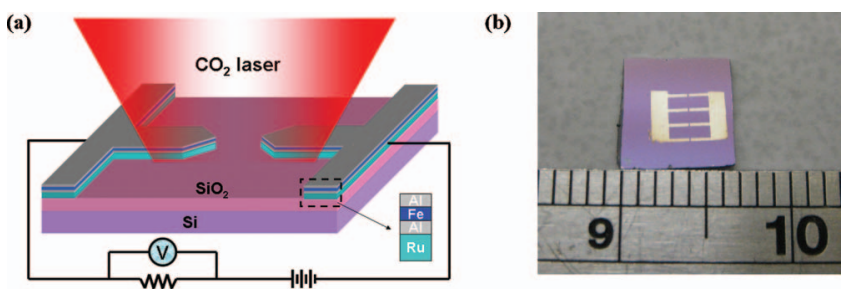

FIG. 1. (Color) Schematic diagram of the LCVD experimental setup (a) and photograph of a patterned substrate (b). 


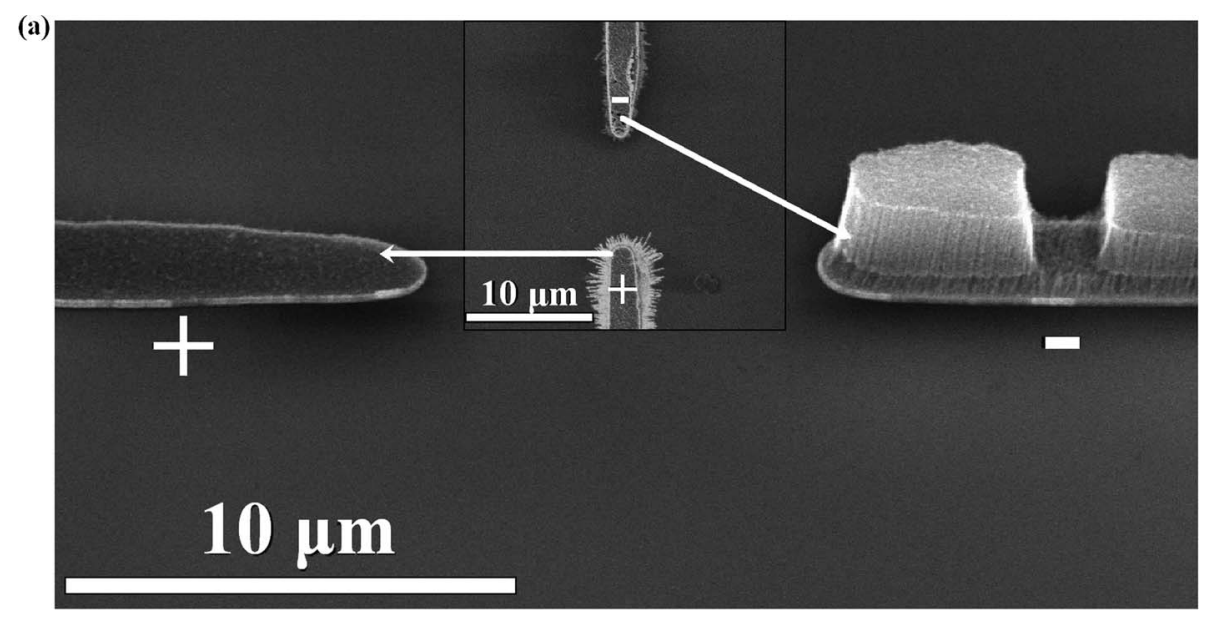

(b)

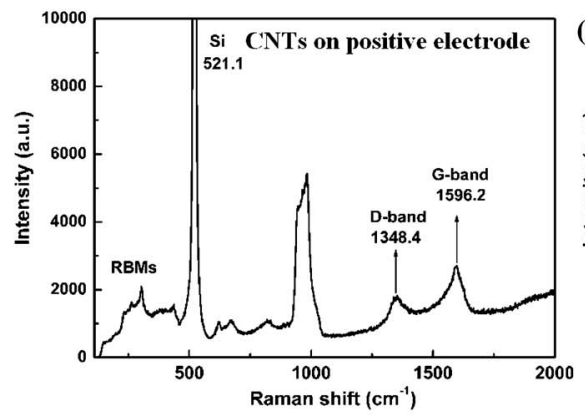

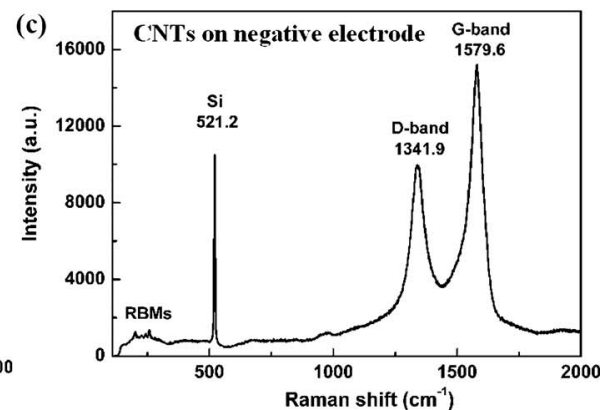

FIG. 2. A typical SEM micrograph of CNTs with different alignments grown on the anode and cathode (a) and typical Raman spectra of CNTs grown on an anode (b) and a cathode (c).
(RBM) signals ranging from 100 to $300 \mathrm{~cm}^{-1},{ }^{18} \mathrm{D}$ and $\mathrm{G}$ bands ${ }^{19,20}$ were observed on both electrodes. The signal observed around $1000 \mathrm{~cm}^{-1}$ was the background noise. The Raman spectrum from the anode shows a stronger RBM intensity while the Raman spectrum from the cathode shows stronger D-band and G-band intensities. The difference in the Raman spectra was ascribed to the different alignments of the CNTs on the electrodes. The surface crawling CNTs on the anode have large Raman scattering cross-sections. Therefore, stronger RBM signals were observed in Fig. 2(b). Vertically aligned CNTs (with end caps exposing to the surface) on the cathode have small Raman scattering cross-sections which lead to weak RBM peaks. The stronger $\mathrm{D}$ and $\mathrm{G}$ bands indicate more carbon deposition, as shown in Fig. 2(c).

To confirm the surface-bounded growth of CNTs on anodes, E-fields of different intensities were applied between the point-to-point electrodes, as shown in Figs. 3(a)-3(d). Fuzzy edges with CNTs sticking out were observed on the anodes. The CNTs grown on the anodes were observed to
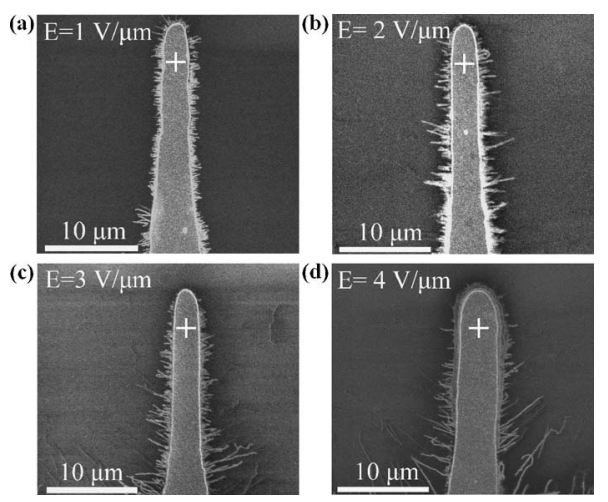

FIG. 3. (a)-(d) are SEM micrographs of surface-bounded CNTs on anodes with different electric fields, $1,2,3$, and $4 \mathrm{~V} / \mu \mathrm{m}$, separately. stretch out from the electrodes and aligned with the E field. For samples growing in the same time period, an increased CNT length was observed for stronger E-field.

A cross-shaped electrode pattern was used to study the growth of CNTs with different alignments influenced by the E-field distributions, as shown in Figs. 4(a)-4(c). The electrodes were numbered from 1 to 4 in a clockwise order starting from the top electrode. In Fig. 4(a), fuzzy edges with crawling CNTs were observed on the anodes 3 and 4 . Some CNTs were observed to stretch out from the cathode 2 , which is ascribed to the formation of the CNT-bridge between the electrodes 2 and 3. Formation of the conductive CNT-bridge between the electrodes 2 and 3 made the two electrodes equipotential, which resulted in the observation of some randomly distributed surfaced-bounded CNTs. In Fig. 4(b), surface-bounded CNTs were observed only on the anodes 3. A very clean edge was observed for the cathode 1 . Randomly distributed surface-bounded CNTs were observed on the electrodes 2 and 4, which were caused by the same reason in Fig. 4(a). In Fig. 4(c), long CNTs were observed between the electrodes 1 and 2. It seems that growth of CNTs on the anode 3 was suppressed compared with the electrodes 2 and 4 due to the weaker $E$ field between the electrode 3 and electrodes 2 and 4.

From the experimental results, it can be concluded that CNT alignments can be controlled by biasing electrodes with
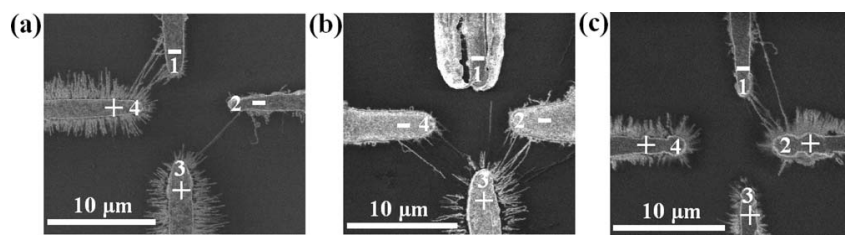

FIG. 4. (a)-(c) are SEM micrographs of CNTs grown on electrodes with different electric field distributions. 

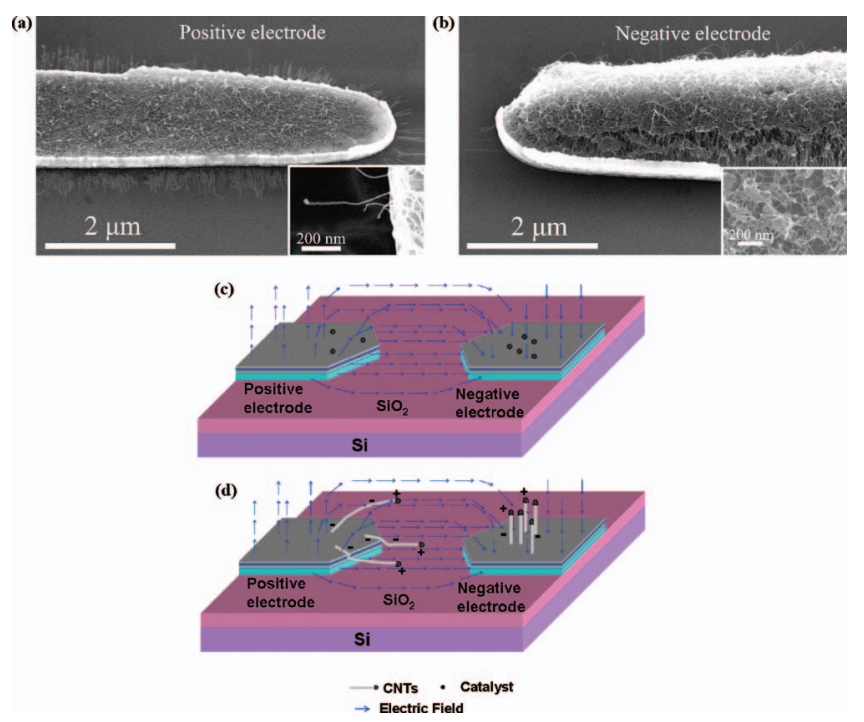

FIG. 5. (Color) (a) and (b) are SEM micrographs of CNTs on the anode and cathode. (c) and (d) are schematic diagrams of CNT growth induced by the movement of positively charged catalyst NPs in the external electric field.

different polarities. Surface-bounded CNTs dominate anodes while vertically aligned CNTs dominate cathodes. CNTs stretch out from the anodes toward the cathodes following the E-field. It was suggested that movement of catalyst NPs was responsible for the CNT alignment. As shown in the inset of the Fig. 5(a), surface crawling CNTs have bright spots at their ends, which are the catalyst NPs. In Fig. 5(b), a shiny top layer was observed for the vertically aligned CNT arrays, which indicates the coalesced catalyst NPs. Therefore, a tip-growth mode is suggested for the CNT growth in the LCVD process. The catalyst Fe NPs are positively charged due to the stronger electron affinity of carbon. Iron has a work function of $4.67 \mathrm{eV}^{21}$ while an average work function of CNTs was calculated to be $5.44 \mathrm{eV}^{22,23}$ Therefore, CNTs have a stronger electron affinity and attract electrons from the iron atoms, making the iron NPs positively charged. As a result, the catalyst NPs are repulsed by the anodes and move away from the anodes toward the cathodes following the E-field. Since CNTs nucleate and grow from carbon-saturated catalyst NPs, CNT growth follows the moving tracks of the catalyst NPs. A stronger E-field would exert stronger force on the catalyst NPs and yield longer aligned CNTs, which supports the observation in Fig. 3. For the CNTs grown on the cathodes, vertically aligned CNTs were ascribed to the joint contribution from the following interactions. The first was the van der Waals interaction among the CNTs, which made high density CNT arrays entangled and vertically aligned. The second was the attractive force between the positively charged catalyst NPs and the cathodes, which prevented CNTs from stretching outside. The third was the repulsing forces caused by the continuous growth of CNTs, which kept pushing the catalyst NPs outward. Therefore, the catalyst NPs were forced to move outside of the surface plane and induced vertically aligned growth of CNTs.

Since $p^{+}-\mathrm{Si}$ substrates were used, a built-in E-field would be formed when a voltage is applied on the electrodes. The built-in E-field would show a contrary potential to the applied voltage. It would also influence the movement of the catalyst NPs. A negative electrical potential was created in the Si substrate underneath the anode, which would attract the positively charged catalyst NPs. While a positive potential was built in the $\mathrm{Si}$ substrate underneath the cathode, which would repulse the positively charged catalyst NPs. Therefore, CNTs stretching out of the anode were observed to be pinned on the substrate surface, while CNTs stretching out of the cathode were forced back to the cathode by the built-in electric field. In addition, there are several other parameters influencing the movement of the catalyst NPs, including gas flow, thermal fluctuation, gas convection, van der Waals force, and physical barrier.

In summary, controllable growth of CNTs of different alignments was achieved by applying biasing voltages of different polarities on metallic electrodes. Surface-bounded CNTs were obtained on anodes. Vertically aligned CNTs were obtained on cathodes. The growth alignment control was ascribed to the movement of positively charged catalyst NPs. By applying the biasing induced alignment control, CNTs of different alignments could be easily obtained for applications of different fields.

The authors are grateful to the National Science Foundation for the financial support (Grant Nos. ECCS 0621899 and ECCS 0652905).

${ }^{1}$ J. Shi, Y. F. Lu, K. J. Yi, Y. S. Lin, S. H. Liou, J. B. Hou, and X. W. Wang, Appl. Phys. Lett. 89, 083105 (2006).

${ }^{2}$ W. Xiong, Y. S. Zhou, M. Mahjouri-Samani, W. Q. Yang, K. J. Yi, X. N. He, S. H. Liou, and Y. F. Lu, Nanotechnology 20, 025601 (2009).

${ }^{3}$ Y. G. Zhang, A. Chang, J. Cao, Q. Wang, W. Kim, Y. M. Li, N. Morris, E. Yenilmez, J. Kong, and H. J. Dai, Appl. Phys. Lett. 79, 3155 (2001).

${ }^{4}$ C. J. Lee, D. W. Kim, T. J. Lee, Y. C. Choi, Y. S. Park, Y. H. Lee, W. B. Choi, N. S. Lee, G. S. Park, and J. M. Kim, Chem. Phys. Lett. 312, 461 (1999).

${ }^{5}$ S. Fan, M. G. Chapline, N. R. Franklin, T. W. Tombler, A. M. Cassell, and H. Dai, Science 283, 512 (1999).

${ }^{6}$ G. L. Che, B. B. Lakshmi, E. R. Fisher, and C. R. Martin, Nature (London) 393, 346 (1998).

${ }^{7}$ Y. T. Liu, X. M. Xie, Y. F. Gao, Q. P. Feng, L. R. Guo, X. H. Wang, and X. Y. Ye, Mater. Lett. 61, 344 (2007).

${ }^{8}$ S. M. Huang, M. Woodson, R. Smalley, and J. Liu, Nano Lett. 4, 1025 (2004).

${ }^{9}$ Y. Avigal and R. Kalish, Appl. Phys. Lett. 78, 2291 (2001).

${ }^{10}$ C. Bower, W. Zhu, S. Jin, and O. Zhou, Appl. Phys. Lett. 77, 830 (2000)

${ }^{11}$ M. Chhowalla, K. B. K. Teo, C. Ducati, N. L. Rupesinghe, G. A. J. Amaratunga, A. C. Ferrari, D. Roy, J. Robertson, and W. I. Milne, J. Appl. Phys. 90, 5308 (2001).

${ }^{12}$ Q. Yang, C. Xiao, W. Chen, A. K. Singh, T. Asai, and A. Hirose, Diamond Relat. Mater. 12, 1482 (2003).

${ }^{13}$ A. Srivastava, A. K. Srivastava, and O. N. Srivastava, Carbon 39, 201 (2001).

${ }^{14}$ W. R. L. Vander, T. M. Ticich, and V. E. Curtis, Chem. Phys. Lett. 323, 217 (2000).

${ }^{15}$ Q. L. Bao and C. X. Pan, Nanotechnology 17, 1016 (2006).

${ }^{16}$ A. Nojeh, A. Ural, R. F. Pease, and H. J. Dai, J. Vac. Sci. Technol. B 22 3421 (2004).

${ }^{17}$ T. K. Jang, J. H. Ahn, Y. H. Lee, and B. K. Ju, Chem. Phys. Lett. 372, 745 (2003).

${ }^{18}$ S. Dresselhaus, M. G. Dresselhaus, R. Saito, and A. Jorio, Phys. Rep. 409, 47 (2005).

${ }^{19}$ J. Yu, Q. Zhang, J. Ahn, S. F. Yoon, Y. J. Li Rusli, B. Gan, K. Chew, and K. H. Tan, Diamond Relat. Mater. 10, 2157 (2001).

${ }^{20}$ Y. M. Shyu and F. C. N. Hong, Mater. Chem. Phys. 72, 223 (2001).

${ }^{21}$ CRC Handbook of Chemistry and Physics, 85th ed. (CRC, Boca Raton, 2005).

${ }^{22}$ G. Zhou, W. Duan, and B. Gu, Phys. Rev. Lett. 87, 095504 (2001).

${ }^{23}$ Q. K. Yu, G. T. Qin, H. Li, Z. H. Xia, Y. B. Nian, and S. S. Pei, J. Phys. Chem. B 110, 22676 (2006). 